\title{
Unravelling the role of quantum interference in the weak-field laser phase modulation control of photofragment distributions
}

\section{García-Vela, Alberto; Henriksen, Niels Engholm}

Published in:

Physical Chemistry Chemical Physics

Link to article, DOI:

$10.1039 / \mathrm{c} 5 \mathrm{cp} 06094 \mathrm{j}$

Publication date:

2016

Document Version

Peer reviewed version

Link back to DTU Orbit

Citation (APA):

García-Vela, A., \& Henriksen, N. E. (2016). Unravelling the role of quantum interference in the weak-field laser phase modulation control of photofragment distributions. Physical Chemistry Chemical Physics, 18(6), 47724779. https://doi.org/10.1039/c5cp06094j

\section{General rights}

Copyright and moral rights for the publications made accessible in the public portal are retained by the authors and/or other copyright owners and it is a condition of accessing publications that users recognise and abide by the legal requirements associated with these rights.

- Users may download and print one copy of any publication from the public portal for the purpose of private study or research.

- You may not further distribute the material or use it for any profit-making activity or commercial gain

- You may freely distribute the URL identifying the publication in the public portal 


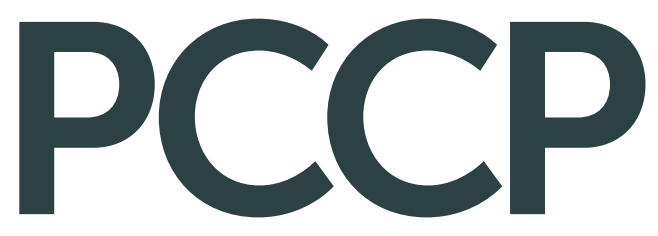

Accepted Manuscript

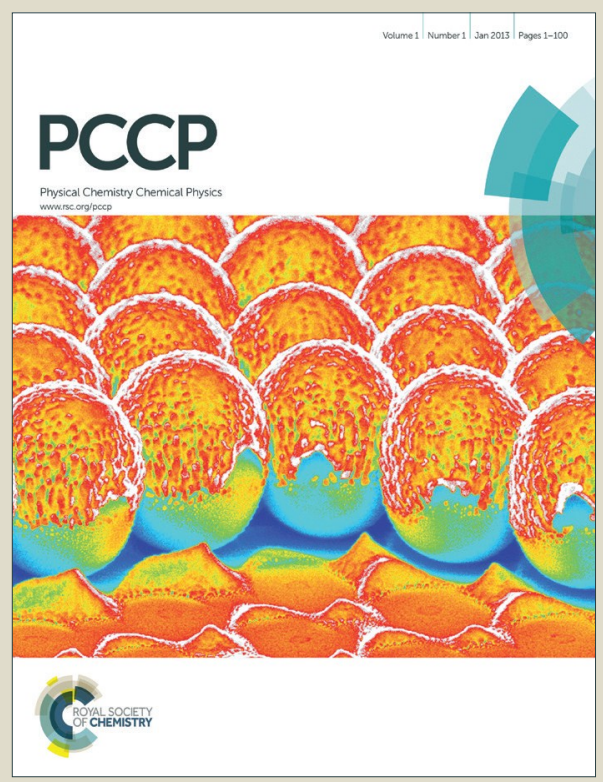

This is an Accepted Manuscript, which has been through the Royal Society of Chemistry peer review process and has been accepted for publication.

Accepted Manuscripts are published online shortly after acceptance, before technical editing, formatting and proof reading. Using this free service, authors can make their results available to the community, in citable form, before we publish the edited article. We will replace this Accepted Manuscript with the edited and formatted Advance Article as soon as it is available.

You can find more information about Accepted Manuscripts in the Information for Authors.

Please note that technical editing may introduce minor changes to the text and/or graphics, which may alter content. The journal's standard Terms \& Conditions and the Ethical guidelines still apply. In no event shall the Royal Society of Chemistry be held responsible for any errors or omissions in this Accepted Manuscript or any consequences arising from the use of any information it contains. 


\title{
Unravelling the role of quantum interference in the weak-field laser phase modulation control of photofragment distributions
}

\author{
Alberto García-Vela*,1 and Niels E. Henriksen ${ }^{2}$ \\ ${ }^{1}$ Instituto de Física Fundamental, Consejo Superior de Investigaciones Científicas, \\ Serrano 123, 28006 Madrid, Spain \\ ${ }^{2}$ Department of Chemistry, Building 207, Technical University of Denmark, \\ DK-2800 Kgs. Lyngby, Denmark
}

\begin{abstract}
The role played by quantum interference in the laser phase modulation coherent control of photofragment distributions in the weak-field regime is investigated in detail in this work. The specific application involves realistic wave packet calculations of the transient vibrational populations of the $\operatorname{Br}_{2}\left(B, v_{f}\right)$ fragment produced upon predissociation of the Ne- $\mathrm{Br}_{2}(B)$ complex, which is excited to a superposition of overlapping resonance states using different fixed bandwidth pulses where the linear chirps are varied. The postpulse transient phase modulation effects found on the fragment populations for a long time window are explained in terms of the mechanism of interference between the overlapping resonances. A detailed description of how the interference mechanism affects the magnitude and the time window of the phase control effects is also provided. In the light of the results, the conditions to maximize phase modulation control on the fragment distributions are discussed.
\end{abstract}

*Corresponding author. E-mail: garciavela@iff.csic.es 


\section{Introduction}

Coherent control of a molecular process aimed at steering its dynamical evolution towards a desired target has been a goal pursued for a long time ${ }^{1,2}$. This type of control relies both on the coherent nature of light and on the quantum nature of light and matter. Control strategies have been successfully designed and applied experimentally to different molecular processes within both weak-field and strongfield conditions. ${ }^{3-14}$ From the theoretical point of view, a variety of control schemes has also been proposed. ${ }^{1,2,15-26}$ However, despite the spectacular advances occurred in the last years in the field of coherent control, still many challenges and questions remain open.

In addition to the control effects achieved by varying the frequency bandwidth and the intensity of the laser pulse used, the influence of modulating the laser phase on the control effects has been extensively investigated in the last years. More specifically, the questions about how and to what extent control can be exerted by varying the phase dependence of a fixed bandwidth laser pulse in the weak-field, one-photon limit, have been addressed. Weak-field coherent control (vs strong-field control) has the advantage of preserving the inherent dynamics of the system. In this sense, a most relevant development was the theoretical proof that for closed systems initially in an eigenstate of the electronic ground state, phase control of the asymptotic, long-time limit final state distributions of photodissociation fragments is not possible in the weak-field regime. ${ }^{27,28}$ Indeed, that proof showed that asymptotic product distributions are independent on the laser phase, and thus phase modulation cause no effects on the final fragment distributions. ${ }^{27-29}$ Later on, an experiment on weak-field coherent control of retinal isomerization was reported, where longtime control effects of the isomerization yields were observed by modulating the laser phase. ${ }^{6}$ This finding appeared to contradict the previous theoretical proof. In order to clarify this controversy, the conditions for one-photon coherent phase control in isolated and open quantum systems were established. ${ }^{30}$ In the light of these conditions, it was concluded that the modes involved in retinal isomerization 
can be considered as a subsystem coupled intramolecularly to the environment of the remaining modes of this large molecule, and under these "open" system conditions, weak-field coherent phase control of the subsystem properties is possible.

However, transient postpulse one-photon coherent phase control has been demonstrated in the photodissociation of diatomic molecules. Thus, after the laser pulse vanished it was, e.g., observed that the total dissociation probability as well as the branching ratio between different electronic states could be modulated by the spectral phases. ${ }^{24-26}$ Furthermore, it has been shown that when a superposition of nonorthogonal, interfering states, like overlapping resonances, is prepared, transient weak-field phase control of the time-dependent populations of some electronic states in different systems is possible during a postpulse time window. ${ }^{24,31}$ More recently it was reported the first computational demonstration of coherent control of the final state distribution of photodissociation fragments based on pure phase modulation of the pump pulse in the weak-field regime. ${ }^{32}$ The specific process investigated was the

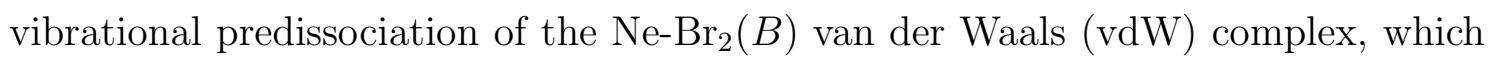
is excited to a superposition of overlapping resonance states using fixed bandwidth pulses with different linear chirps. Phase control effects on the transient vibrational populations of the $\operatorname{Br}_{2}\left(B, v_{f}\right)$ fragment produced upon predissociation were demonstrated by varying the chirp rate, and such control effects were found to persist for a long-time window of about 200 ps after the pulse is over.

The postpulse long-time window of phase control effects on the $\operatorname{Br}_{2}\left(B, v_{f}\right)$ fragment populations was attributed to the change of the mechanism of interference between the $\mathrm{Ne}^{-} \mathrm{Br}_{2}(B)$ overlapping resonances when the chirp rate varies. ${ }^{32}$ When a coherent superposition of nonoverlapping (and therefore noninterfering) $\mathrm{Ne}-\mathrm{Br}_{2}(B)$ resonances was prepared, phase effects on the $\operatorname{Br}_{2}\left(B, v_{f}\right)$ populations by varying the chirp rate were found only during the time that the pump pulse was on, and this absence of postpulse control effects supported the above conclusion. Thus, it would be most interesting to understand in detail how modulation of the laser pulse phase affects interference between the overlapping resonances, and how this is reflected in 
the final control effects on the transient fragment populations, and this is the aim of the present work. Such a detailed understanding would provide the possibility to exert a more active control by manipulating the interference mechanism, in order to increase the intensity of the phase effects and the size of the postpulse time window, or even to change the specific times when the phase effects occur in the populations.

This paper is organized as follows. Section II presents a description of the main features of the methodology applied and the computational details. In section III the results are presented and discussed. Conclusions are given in section IV.

\section{Methodology}

In our previous work ${ }^{32}$ we considered the fragmentation of the $\mathrm{Ne}-\mathrm{Br}_{2}$ van der

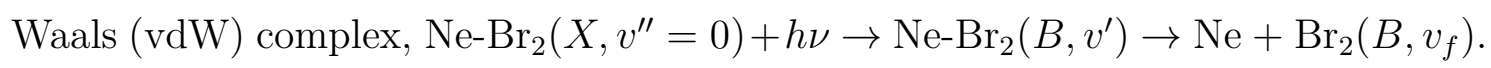
The dissociation dynamics of this complex has been studied in much detail, ${ }^{33-37}$ and it has been shown that the dynamics in some energy ranges encompass overlapping resonances. ${ }^{34-36}$ Interference between these overlapping resonances of Ne$\mathrm{Br}_{2}(B)$ have been used to design schemes to control the lifetime of specific resonance states. $^{22,23,38}$ The signature of interference between resonances on the transient fragment state distributions has been highlighted previously when a superposition of resonances is created by a pulse with a sufficiently large bandwidth. ${ }^{39}$ Recent work on coherent control has discussed the significant role of interference between overlapping resonances in relation to coherent phase control. ${ }^{31,32,40}$

Specifically, the $\mathrm{Ne}_{-} \mathrm{Br}_{2}\left(B, v^{\prime}=27\right)$ complex was previously studied ${ }^{32}$ because its ground intermolecular resonance overlaps with some vdW orbiting resonances located in the lower $v^{\prime}-1$ vibrational manifold of $\operatorname{Br}_{2}(B) .{ }^{36,37}$ These orbiting resonances lie above the $\mathrm{Ne}+\mathrm{Br}_{2}\left(B, v^{\prime}-1=26, j^{\prime}=0\right)$ dissociation threshold, and are supported by centrifugal barriers. In particular, the $v^{\prime}$ ground resonance overlaps mainly with a $v^{\prime}-1$ orbiting resonance located $\sim 1.2 \mathrm{~cm}^{-1}$ above in energy. The excitation spectrum of the $\mathrm{Ne}_{-} \mathrm{Br}_{2}\left(B, v^{\prime}=27\right)$ ground resonance (see Fig. 2 of ref. 32) displays a main peak located at $-61.80 \mathrm{~cm}^{-1}$, associated with the $v^{\prime}$ ground 
vdW resonance, and several other overlapping peaks associated with $v^{\prime}-1$ orbiting resonances. The second most intense feature in the spectrum, located at -60.63 $\mathrm{cm}^{-1}$, corresponds to the $v^{\prime}-1$ orbiting resonance separated from the $v^{\prime}$ ground resonance by $1.2 \mathrm{~cm}^{-1}$. Thus, $\mathrm{Ne}_{-} \mathrm{Br}_{2}\left(B, v^{\prime}=27\right)$ provides the simplest case of overlapping resonances, namely essentially only two resonances (those located at -61.80 and $-60.63 \mathrm{~cm}^{-1}$ ), which makes easier the analysis of the mechanism of interference between them, and its effect on the phase coherent control.

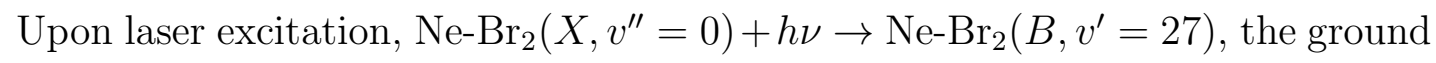
intermolecular resonance of $\mathrm{Ne}-\mathrm{Br}_{2}\left(B, v^{\prime}=27\right)$, along with other overlapping resonances, are populated. Then the resonances excited decay to the fragmentation continuum through vibrational predissociation, $\mathrm{Ne}_{-} \mathrm{Br}_{2}\left(B, v_{i}\right) \rightarrow \mathrm{Ne}+\mathrm{Br}_{2}\left(B, v_{f}<v^{\prime}\right)$, being $v_{i}=v^{\prime}, v^{\prime}-1 .^{33-36}$ The process of $\mathrm{Ne}^{-\mathrm{Br}_{2}}\left(B, v_{i}\right)$ excitation with a laser pulse and the subsequent predissociation of the complex was simulated with a full threedimensional wave packet method (assuming $J=0$ ) described in detail elsewhere. ${ }^{22,35}$ It is noted that the lifetime calculated with the present theoretical model for the decay of the $\mathrm{Ne}_{-} \mathrm{Br}_{2}\left(B, v^{\prime}=16\right)$ ground intermolecular resonance has been found to be $69 \mathrm{ps}^{41}{ }^{41}$ while the corresponding lifetime estimated experimentally is $68 \pm 3 \mathrm{ps}^{42}$ This good agreement with the experimental lifetime implies that both the threedimensional wave packet method and the potential surfaces used in the present simulations are quite realistic to describe this resonance decay process.

In order to excite the $\mathrm{Ne}_{-} \mathrm{Br}_{2}\left(B, v^{\prime}=27\right)$ ground resonance, we consider a pump pulse with a fixed Gaussian frequency distribution centered around $\omega_{0}$ (which is the frequency required for excitation of the $v^{\prime}=27$ ground intermolecular resonance), including a quadratic spectral phase function with chirp parameter $\beta_{0}$. The electric

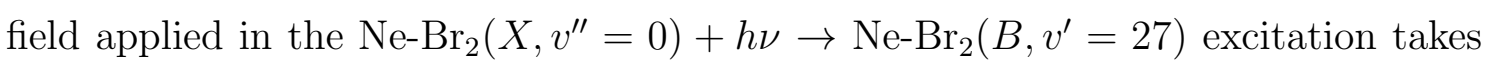
the form

$$
\mathcal{E}(t)=\mathcal{E}_{0} \operatorname{Re}\left[\sqrt{\frac{\tau_{0}^{2}}{\tau_{0}^{2}-i \beta_{0}}} \exp \left(-\frac{\left(t-t_{0}\right)^{2}}{2 \tau^{2}}-\frac{i \beta\left(t-t_{0}\right)^{2}}{2}-i \omega_{0}\left(t-t_{0}\right)\right)\right]
$$


with a linear chirp rate $\beta$ and a pulse duration $\tau$ related to $\tau_{0}$ and $\beta_{0}$ via

$$
\beta=\frac{\beta_{0}}{\left(\tau_{0}^{4}+\beta_{0}^{2}\right)},
$$

and

$$
\tau^{2}=\tau_{0}^{2}\left(1+\frac{\beta_{0}^{2}}{\tau_{0}^{4}}\right)
$$

In the work of ref. 32 the value $\tau_{0}=2.12 \mathrm{ps}$ was used in the above equations. In this case the spectral full width at half-maximum (FWHM) associated with the pulse is $4 \mathrm{~cm}^{-1}$, and the full bandwidth of the pulse covers $\sim 14 \mathrm{~cm}^{-1}$. For a transformlimited pulse $\left(\beta_{0}=\beta=0\right)$ the above value of $\tau_{0}$ corresponds to a temporal FWHM of the pulse of $\tau_{\text {FWHM }}=\tau \sqrt{8 \ln 2}=\tau_{0} \sqrt{8 \ln 2}=5$ ps. The laser pulse is assumed to be linearly polarized. The transition dipole moment function for the $X \rightarrow B$ transition is unknown, so it was assumed to be constant (1 atomic unit).

As qualitatively discussed in ref. 32, the effect of varying the chirp rate is essentially to modify the time at which each resonance of the superposition is populated, which modifies correspondingly the way that the different resonances interfere between themselves along time, causing changes in the $\operatorname{Br}_{2}\left(B, v_{f}\right)$ fragment populations. In this work the aim is to analyze in detail how interference between the overlapping resonances affects the coherent phase control. To this purpose, a convenient strategy is to investigate how the control effects achieved on the $\operatorname{Br}_{2}\left(B, v_{f}\right)$ fragment populations by varying the chirp rate, change when the conditions in which interference between the overlapping resonances occurs are modified. A simple way to change the conditions of occurrence of interference is to vary the temporal width of the unchirped pump pulse, which will vary correspondingly the bandwidth of the pulse. Indeed, by modifying the bandwidth of the pump pulse the relative amplitudes of the different overlapping resonances in the coherent superposition created are also modified ${ }^{22}$, leading to a different interference pattern.

Thus, in the simulations reported here pump pulses with four different temporal widths have been used, namely $\tau_{\mathrm{FWHM}}=40,10,5$, and 2.5 ps. The spectral FWHM of the above pulses is $0.5,2,4$, and $8 \mathrm{~cm}^{-1}$, respectively, and their full bandwidth covers $\sim 2, \sim 7, \sim 14$, and $\sim 28 \mathrm{~cm}^{-1}$, respectively. It is noted that even in 
the case of the largest bandwidth pulse, only the ground intermolecular resonance is populated in the $v^{\prime}=27$ vibrational manifold, since the first excited resonance in this manifold is separated from the ground one by about $18 \mathrm{~cm}^{-1}$, which is more than half the bandwidth of the FWHM=2.5 ps pulse. All the pulses have a maximum amplitude of $\mathcal{E}_{0}=1.0 \times 10^{-6}$ a.u., which corresponds to a maximum pulse intensity of about $3.5 \times 10^{4} \mathrm{~W} / \mathrm{cm}^{2}$, well within the weak-field regime. The same central frequency $\omega_{0}$ required to excite the $\mathrm{Ne}_{-} \mathrm{Br}_{2}\left(B, v^{\prime}=27\right)$ ground intermolecular resonance was used in all the pulses applied. For each pulse the values $\beta_{0}=0,12.0$, 24.0, 50.0, and $75.0 \mathrm{ps}^{2}$ for the chirp rate are used.

\section{Results and discussion}

In Fig. 1 the Gaussian envelopes of the four pump pulses used in the simulations are shown for the different values of $\beta_{0}$, showing the temporal range covered by the pulses as the chirp rate increases. The value of the chirp rate $\beta$ is determined by eqn (2), and when $\tau_{0}$ (directly related to $\tau_{\text {FWHM }}$ ) is large it dominates the denominator of eqn (2) and causes a small dependence of $\beta$ on the value of $\beta_{0}$. As a result, for $\tau_{\mathrm{FWHM}}=40 \mathrm{ps}$ the profile of the pump pulse has a really small dependence on the chirp rate, so practically no phase control effects on the $\operatorname{Br}_{2}\left(B, v_{f}\right)$ vibrational populations should be expected by using these pulses. As $\tau_{\text {FWHM }}$ decreases the chirp rate dependence of the pulse profile increases. For $\tau_{\mathrm{FWHM}}=5$ and 2.5 ps the chirp rate dependence of the pulse shape is maximized and those pulse profiles display the largest temporal spreadings. These pulses are expected to induce the largest phase control effects on the transient fragment populations.

As discussed above, phase coherent control effects on the transient $\operatorname{Br}_{2}\left(B, v_{f}\right)$ fragment populations are the result of the interaction of (i) the mechanism of interference between the overlapping resonances populated in the superposition created, with (ii) the modulation of the pulse phase achieved by varying the pulse chirp rate. Thus, by establishing the correlation between the combined changes of both the interference mechanism and the laser pulse phase, and the final effects on the 
fragment populations, we will be able to determine the role played by interference in the phase control effects achieved. The profiles of Fig. 1 provide an indication of the extent of the changes in the pulse phase for the four different temporal widths. We now need a similar determination of the extent of the changes in the interference mechanism in combination with the phase modulation of the pulse for the different temporal widths, in order to establish the above correlation.

It has been previously found that the survival probability of one of the interfering resonances provides a sensitive probe of the magnitude of interference between the overlapping resonances. ${ }^{22,23,38}$ Indeed, we can express the coherent superposition of

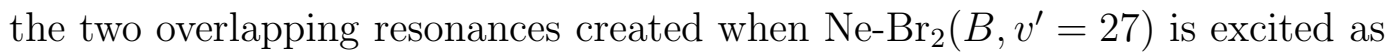

$$
\Phi(t)=a_{1}(t) \psi_{1}(t)+a_{2}(t) \psi_{2}(t)
$$

where $\psi_{1}(t)$ and $\psi_{2}(t)$ are the zeroth-order $v^{\prime}$ ground resonance and the zeroth-order $v^{\prime}-1$ orbiting resonance, respectively. Then the survival probability associated with the $\psi_{1}$ resonance can be expressed as

$$
\begin{gathered}
I_{1}(t)=\left|<\psi_{1}\right| \Phi(t)>\left.\right|^{2}=\left|a_{1}(t)<\psi_{1}\right| \psi_{1}(t)>+a_{2}(t)<\psi_{1}\left|\psi_{2}(t)>\right|^{2}= \\
\left|a_{1}(t)\right|^{2}\left|<\psi_{1}\right| \psi_{1}(t)>\left.\right|^{2}+\left|a_{2}(t)\right|^{2}\left|<\psi_{1}\right| \psi_{2}(t)>\left.\right|^{2}+ \\
a_{1}(t) a_{2}(t)^{*}<\psi_{1}\left|\psi_{1}(t)><\psi_{2}(t)\right| \psi_{1}>+a_{1}(t)^{*} a_{2}(t)<\psi_{1}(t)\left|\psi_{1}><\psi_{1}\right| \psi_{2}(t)>,
\end{gathered}
$$

and a similar equation holds for $I_{2}(t)=\left|<\psi_{2}\right| \Phi(t)>\left.\right|^{2}$. The $\psi_{1}$ wave function is calculated variationally, as previously described in detail. ${ }^{22,35}$ The specific preparation of the superposition $\Phi(t)$ determines the values of the amplitude coefficients $a_{1}(t)$ and $a_{2}(t)$, and these coefficients determine the intensity of the last three terms of the right-hand side of eqn (5), which are the interference terms of the survival probability. This is the reason why $I_{1}(t)$ (or $I_{2}(t)$ ) gives a sensitive measure of the intensity of interference between the overlapping resonances.

In Fig. 2 the survival probabilities $I_{1}(t)$ obtained when the four pump pulses are used to prepare the coherent superposition of overlapping resonances are shown for the different values of $\beta_{0}$. The main result of Fig. 2 is that the magnitude of the 
structure of undulations associated with interference between the two resonances becomes increasingly more pronounced as the spectral width of the pump pulse increases. The reason is that by gradually increasing the bandwidth of the pulse, an increase of the $a_{2}(t)$ coefficient in the $\Phi(t)$ superposition is achieved, leading to an increase of the intensity of interference between the resonances (see eqn (5)). For $\mathrm{FWHM}=40 \mathrm{ps}$ the pulse bandwidth is very narrow, and essentially only the $\psi_{1}$ resonance is populated (i.e., $a_{2}(t)$ is very small). In this situation interference with $\psi_{2}$ is very low, and the $I_{1}(t)$ survival probability corresponds to a single isolated $\psi_{1}$ resonance with no undulations. In the practical absence of interference variation of the chirp rate has no effect, and the survival probabilities for the different $\beta_{0}$ values are practically coincident. As the pulse bandwidth increases the $\psi_{2}$ resonance begins to be significantly populated and the onset of interference appears in the form of the structure of undulations. The intensity of this structure increases with increasing bandwidth, as the $a_{2}(t)$ coefficient grows. As should be expected, an increasing intensity of interference leads to increasingly different $I_{1}(t)$ curves when the chirp rate is varied, as seen in the panels corresponding to $\mathrm{FWHM}=10,5$, and $2.5 \mathrm{ps}$. Indeed, the more intense is the mechanism of interference, the larger will be the effects of changing that mechanism when the chirp rate is varied.

Now, Fig. 3 shows the time-dependent normalized ${ }^{32} \operatorname{Br}_{2}\left(B, v_{f}=v^{\prime}-1\right)$ fragment vibrational populations calculated with the four pump pulses for the different values of $\beta_{0}$. Similarly as in the curves of Fig. 2, the intensity of the effects of varying the chirp rate on the fragment populations increases with increasing pulse bandwidth, i.e., with increasing intensity of interference. Thus, Figs. 2 and 3 clearly establish the correlation between the intensity of the mechanism of interference between the overlapping resonances and the intensity of the phase effects achieved on the fragment populations when the chirp rate of the pulse is modified. Enhancement of the intensity of interference leads to an enhancement of the phase effects on the populations.

At this point it is interesting to analyze in more detail the specific role played 
by interference in the transient phase effects displayed by the fragment populations. More specifically, we wish to address questions like why the transient phase effects on the population curves appear at the same specific fixed times regardless of the time duration of the pulse applied; why the intensity of the effects is gradually damped with increasing time, leading to an absence of phase modulation effects in the asymptotic limit; and how the intensity and the time window of the phase effects on the product populations can be maximized. In order to answer those questions we will analyze the behavior of both the $\operatorname{Br}_{2}\left(B, v_{f}=v^{\prime}-1\right)$ fragment populations and the $I_{1}(t)$ survival probabilities in the time range $t>100 \mathrm{ps}$, where the phase effects on the populations occur at the same specific times for the different pump pulses. Such curves are shown in Figs. 4-6 for the FWHM=10, 5, and 2.5 ps pulses, respectively.

The $v^{\prime}-1$ population curves of Figs. 4a, 5a, and 6a show that the main phase modulation effects occur at the same specific times, that can be identified as $t \sim 150$, $\sim 200, \sim 240, \sim 300, \sim 380$, and $\sim 450$ ps. Taking into account that the maximum half duration of the FWHM=2.5 ps pulse with $\beta_{0}=75 \mathrm{ps}^{2}$ is $200 \mathrm{ps}$, and that all the other pulses used have a maximum half duration $<150$ ps (see Fig. 1), in most cases the above phase effects occur at times after the pump pulse is over. Therefore, the transient phase effects are postpulse effects, and the specific times at which they take place are essentially independent on the temporal width of the pulse applied.

The above behavior can be understood by examining the survival probabilities of the lower panels of Figs. 4-6. Actually, all those curves display a very similar pattern of undulations. Such a pattern is determined by the specific mechanism of interference between the two overlapping resonances, which is inherent to the resonances. Modifying the bandwidth of the pump pulse leads to quantitative changes in the pattern, due to the change produced in the $a_{1}(t)$ and $a_{2}(t)$ coefficients, but the qualitative overall shape of the pattern remains nearly the same. In other words, by increasing the bandwidth of the pulse, which increases the intensity of interference, an increasingly reacher structure of narrow peaks gradually appears superimposed 
on the overall structure of broad bumps. In this sense, it is noted that for the pulse with $\mathrm{FWHM}=40 \mathrm{ps}$ and $\beta_{0}=0 \mathrm{ps}^{2}$, the same overall structure of broad bumps located at the same times as those of Figs. 4-6 is found, albeit much smoother. A smooth overall structure of broad bumps is the signature of weak interference between the resonances, and a richer structure of narrow peaks appears superimposed on the overall pattern as the intensity of interference increases.

Not surprisingly, the time positions of the transient phase effects in the fragment populations coincide with the positions of the broad bumps of the overall structure of the survival probabilities. Actually these bumps are the signature of interference between the resonances, and interference is the requirement to achieve phase effects in the populations, as discussed above. The interesting result shown by the survival probabilities of Figs. 4-6 is that, as the intensity of interference inceases giving rise to a denser structure of narrow peaks in the curves, an increasing dephasing in the time positions of the peaks (in the sense of increasing differences in the peak positions) appears when the chirp rate is modified. Such a dephasing of the peak positions is the origin of the differences displayed at fixed times by the fragment populations when the chirp rate is varied, i.e., the transient phase effects. The dephasing is caused by changes in the mechanism of resonance interference produced by modifying the phase of the laser pulse. The larger is the dephasing the more intense will be the phase effect produced in the fragment populations, as shown by Figs. 4-6. In the case of the $\mathrm{FWHM}=40$ ps pulse the survival probabilities obtained when varying the chirp rate in the $t>100$ ps range (not shown) display a very small dephasing of their peak structures, leading to practically negligible phase effetcs in the corresponding fragment populations (see Fig. 3). The increase of the intensity of interference and the appearance of a denser structure of peaks clearly contributes to enhance the dephasing between the different structures when the laser pulse phase is modulated. Thus Figs. 4-6 provide a detailed explanation of the appearance of the phase effects at the same specific time positions, and why the phase effects are enhanced as the interference intensity grows. 
The intensity of the survival probabilities of Figs. 4-6 decays gradually to zero with time. This is the behavior expected from the fact that the interfering resonances decay to the continuum, and once they have decayed, interference between them in the asymptotic region is no longer possible. The gradual vanishing of interference is what causes the transient phase effects on the populations to vanish with time as well, leading to final fragment distributions in the asymptotic time limit which are independent on laser phase modulation in the weak-field regime, as previously proved by the theory. ${ }^{27-29}$

In the light of the above discussion, it is most interesting to try and elucidate under which conditions the intensity of the transient phase effects on the fragment populations can be maximized for a general system. From the present results it appears clear that extending the time window of interference between the overlapping resonances will extend correspondingly the time window of postpulse transient phase effects on the fragment populations. However, both the decay and the interference mechanism of the specific resonances populated in the superposition prepared by the pump pulse are intrinsic properties of those resonances. Extending the time window of the interference mechanism under weak-field conditions, which do not alter the molecular Hamiltonian, is far from being a trivial task, although some efforts in that direction have been previously reported..$^{23,38} \mathrm{~A}$ simple way to maximize the time window of interference is to choose the group of longest-lived overlapping resonances available in the system of interest.

On the other hand, the current findings show that increasing the intensity of interference between the resonances enhance the intensity of the phase effects on the product distributions. There will be an optimal combination of $a_{i}$ coefficients in the superposition of overlapping resonances created that will maximize the intensity of interference between them. Thus, finding or approaching that optimal combination appears to be the simplest way to maximize the magnitude of transient laser phase modulation effects on the fragment populations.

In a similar line of discussion, it is also interesting to comment on the effect that 
increasing the strength of the field applied would have on the interference phase control effects found on the fragment distributions. Increasing the field strength in the weak-field regime implies increasing the amplitudes $a_{1}$ and $a_{2}$ of the resonances in the superposition of Eq. (4) by the same factor. Since a change in the intensity of interference between the resonances requires a change in the relative amplitudes $a_{1}$ and $a_{2}$, and these relative amplitudes do not change by increasing the field strength, such an increase will lead to the same transient normalized vibrational populations. Thus, increasing the field strength in the weak-field regime is not expected to produce additional phase modulation control effects on the fragment distributions. However, as the weak-field regime is left and the strong-field regime is entered by further increasing the field strength, changes in the nature of the Hamiltonian may occur, which will affect the nature itself of the two overlapping resonances. As a consequence, the mechanism of interference between them will be affected qualitatively, albeit in a more unpredictable way.

\section{Conclusions}

Quantum interference between nonorthogonal states, like overlapping resonances populated in a superposition, is a basic requirement to achieve postpulse laser phase modulation coherent control of photofragment product distributions in the weakfield regime. The role played by this interference in the occurrence and magnitude of the phase modulation control effects on the product fragment populations has been investigated in detail in this work. A clear correlation between increasing the intensity of resonance interference by varying the composition of the resonance superposition created, and the enhancement of the magnitude of the phase control effects has been established. More specifically, a detailed description of how the mechanism of interference between resonances originates the transient phase effects on the fragment populations and affects their magnitude, is provided. Based on that description, the conditions under which the degree of phase modulation control on the fragment distributions can be maximized in the weak-field limit, were discussed. 


\section{Acknowledgements}

This work was funded by the Ministerio de Ciencia e Innovación (Spain), Grant No. FIS2011-29596-C02-01, and COST Action program, Grant Nos. CM1401 and CM1405. The Centro de Supercomputación de Galicia (CESGA, Spain) is acknowledged for the use of its resources.

\section{References}

(1) S. A. Rice, M. Zhao, Optical Control of Molecular Dynamics, Wiley, New York, 2000.

(2) M. Shapiro and P. Brumer, Quantum Control of Molecular Processes, Second Edition, Wiley-VCH verlag GmbH and Co. KGaA, Singapore, 2012.

(3) P. Anfinrud, R. de Vivie-Riedle and V. Engel, Proc. Natl. Acad. Sci. USA, 1999, 96, 8328 .

(4) M. Wollenhaupt, A. Assion, D. Liese, C. Sarpe-Tudoran, T. Baumert, S. Zamith, M. A. Boucheme, B. Girard, A. Flettner, U. Weichmann and G. Gerber, Phys. Rev. Lett., 2002, 89, 173001.

(5) C. Daniel, J. Full, L. González, C. Lupulescu, J. Manz, A. Merli, S. Vajda and L. Wöste, Science, 2003, 299, 536.

(6) V. I. Prokhorenko, A. M. Nagy, S. A. Waschuk, L. S. Browun, R. R. Birge and R. J. D. Miller, Science, 2006, 313, 1257.

(7) M. P. A. Branderhorst, P. Londero, P. Wasylczyk, C. Brif, R. L. Kosut, H. Rabitz and I. A. Walmsley, Science, 2008, 320, 638.

(8) C. Ott, A. Kaldun, L. Argenti, P. Raith, K. Meyer, M. Laux, Y. Zhang, A. Blättermann, S. Hagstotz, T. Ding, R. Heck, J. Madroñero, F. Martín and T. Pfeifer, Nature, 2014, 516, 374.

(9) T. C. Weinacht, J. Ahn and P. H. Bucksbaum, Nature, 1999, 397, 233.

(10) R. J. Levis, G. M. Menkir and H. Rabitz, Science, 2001, 292, 709.

(11) E. Skovsen, M. Machholm, T. Ejdrup, J. Thфgersen and H. Stapelfeldt, Phys. Rev. Lett., 2002, 89, 133004. 
(12) B. J. Sussman, D. Townsend, M. I. Ivanov and A. Stolow, Science, 2006, 314, 278.

(13) H. Goto, H. Katsuki, H. Chiba and K. Ohmori, Nat. Phys., 2011, 7, 383.

(14) M. E. Corrales, J. González-Vázquez, G. Balerdi, I. R. Solá, R. de Nalda and L. Bañares, Nat. Chem., 2014, 6, 785.

(15) P. Brumer and M. Shapiro, Chem. Phys. Lett., 1986, 126, 541.

(16) D. J. Tannor, R. Kosloff and S. Rice, J. Chem. Phys., 1986, 85, 5805-5820.

(17) J. D. Farnum, G. Gidofalvi and D. A. Mazziotti, J. Chem. Phys., 2006, 124, 234103.

(18) G. Katz, M. A. Ratner and R. Kosloff, Phys. Rev. Lett., 2007, 98, 203006.

(19) A. K. Tiwari, K. B. M øller and N. E. Henriksen, Phys. Rev. A., 2008, 78, 065402.

(20) G. Katz, M. A. Ratner and R. Kosloff, New J. Phys., 2010, 12, 015003.

(21) O. Atabek, R. Lefebvre, M. Lepers, A. Jaouadi, O. Dulieu, V. Kokoouline, Phys. Rev. Lett., 2011, 106, 173002.

(22) A. García-Vela, J. Chem. Phys., 2012, 136, 134304.

(23) A. García-Vela, J. Phys. Chem. Lett., 2012, 3, 1941.

(24) C. C. Shu and N. E. Henriksen, J. Chem. Phys., 2011, 134, 164308.

(25) C. C. Shu and N. E. Henriksen, J. Chem. Phys., 2012, 136, 044303.

(26) A. K. Tiwari, D. Dey and N. E. Henriksen, Phys. Rev. A, 2014, 89, 023417.

(27) P. Brumer and M. Shapiro, Chem. Phys., 1989, 139, 221.

(28) M. Shapiro and P. Brumer, J. Phys. Chem. A, 2001, 105, 2897.

(29) N. E. Henriksen, Adv. Chem. Phys., 1995, 91, 433.

(30) M. Spanner, C. A. Arango and P. Brumer, J. Chem. Phys., 2010, 133, 151101.

(31) T. Grinev and P. Brumer, J. Chem. Phys., 2014, 140, 124307.

(32) A. García-Vela and N. E. Henriksen, J. Phys. Chem. Lett., 2015, 6, 824.

(33) T. A. Stephenson and N. Halberstadt, J. Chem. Phys., 2000, 112, 2265.

(34) J. A. Cabrera, C. R. Bieler, B. C. Olbricht, W. E. van der Veer and K. C. 
Janda, J. Chem. Phys., 2005, 123, 054311.

(35) A. García-Vela and K. C. Janda, J. Chem. Phys., 2006, 124, 034305.

(36) A. García-Vela, J. Chem. Phys., 2007, 126, 124306.

(37) A. García-Vela, J. Chem. Phys., 2008, 129, 094307.

(38) A. García-Vela, $R S C$ Adv., 2014, 4, 52174.

(39) V. Engel and H. Metiu, J. Chem. Phys., 1990, 92, 2317.

(40) T. Grinev, M. Shapiro and P. Brumer, J. Chem. Phys., 2013, 138, 044306.

(41) A. García-Vela, Phys. Chem. Chem. Phys., 2015, 17, 29072.

(42) M. A. Taylor, J. M. Pio, W. E. van der Veer and K. C. Janda, J. Chem. Phys., 2010, 132, 104309. 


\section{FIGURE CAPTIONS}

Fig. 1 Temporal profiles of the pump pulse of eqn (1) for different values of the temporal FWHM and of the chirp parameter $\beta_{0}$.

Fig. 2 Survival probabilities $I_{1}(t)$ (see eqn (5)) associated with the $\mathrm{Ne}-\mathrm{Br}_{2}\left(B, v^{\prime}=27\right.$ ) ground intermolecular resonance $\left(\psi_{1}\right)$, calculated by using different values of the temporal FWHM for the pump pulses and of the chirp parameter $\beta_{0}$.

Fig. 3 Time-dependent normalized vibrational populations of the $\operatorname{Br}_{2}\left(B, v_{f}=v^{\prime}-1\right)$ fragment produced upon predissociation of $\mathrm{Ne}_{-} \mathrm{Br}_{2}\left(B, v^{\prime}=27\right)$, calculated by using different values of the temporal FWHM for the pump pulses and of the chirp parameter $\beta_{0}$.

Fig. 4 (a) Time-dependent normalized vibrational populations of the $\operatorname{Br}_{2}\left(B, v_{f}=v^{\prime}-\right.$ 1) fragment produced upon predissociation of $\operatorname{Ne}_{-} \mathrm{Br}_{2}\left(B, v^{\prime}=27\right)$, calculated with the pump pulse with $\mathrm{FWHM}=10 \mathrm{ps}$ for different values of the chirp parameter $\beta_{0}$. (b) Survival probabilities $I_{1}(t)$ calculated with the same pump pulse and values of the chirp parameter $\beta_{0}$ as in (a). For the sake of clarity, the curves corresponding to only three values of $\beta_{0}$ have been included in the figure.

Fig. 5 Same as Fig. 4 but for the pump pulse with FWHM= 5 ps.

Fig. 6 Same as Fig. 4 but for the pump pulse with $\mathrm{FWHM}=2.5$ ps. 
Fig. 1

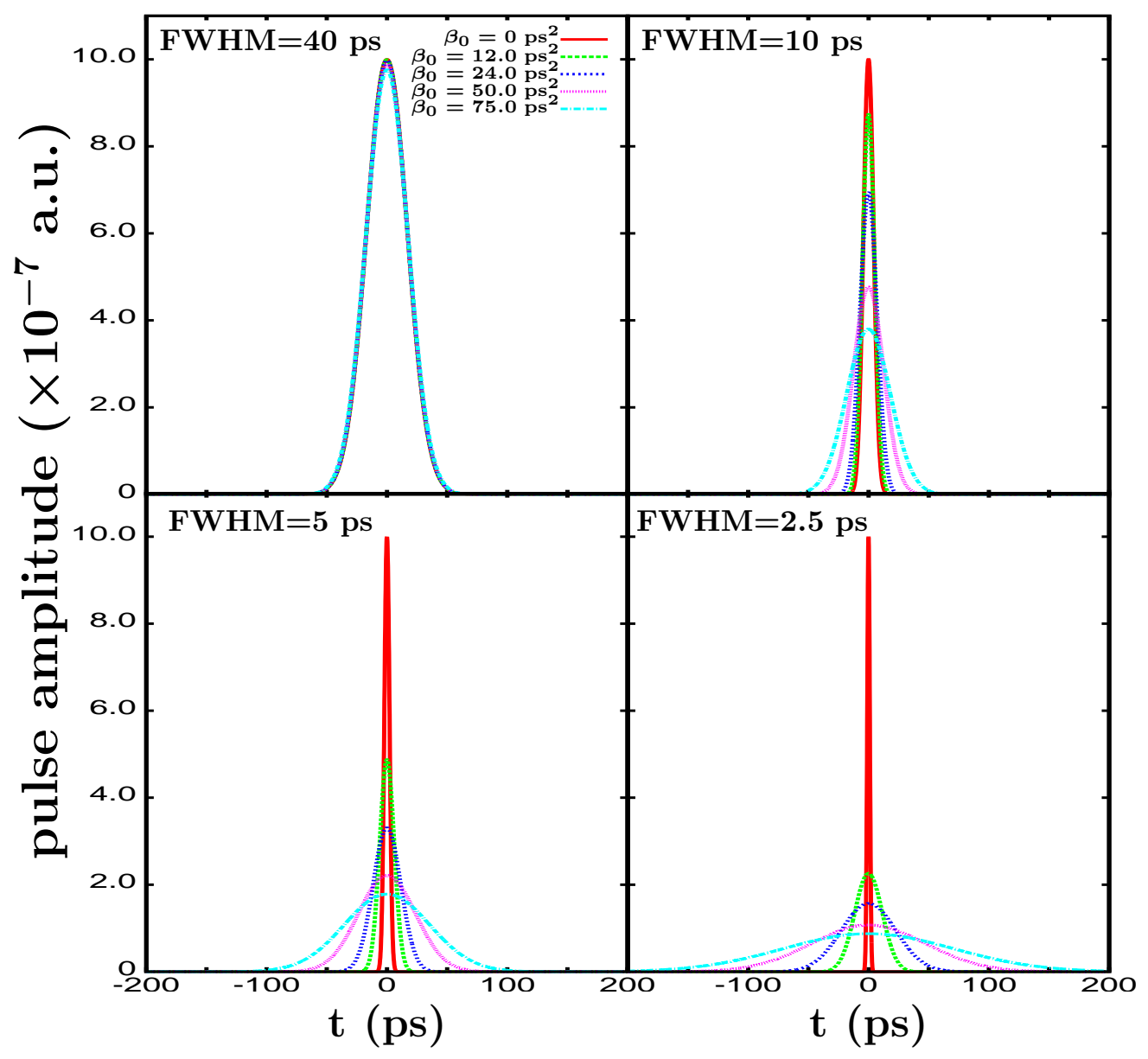


Fig. 2

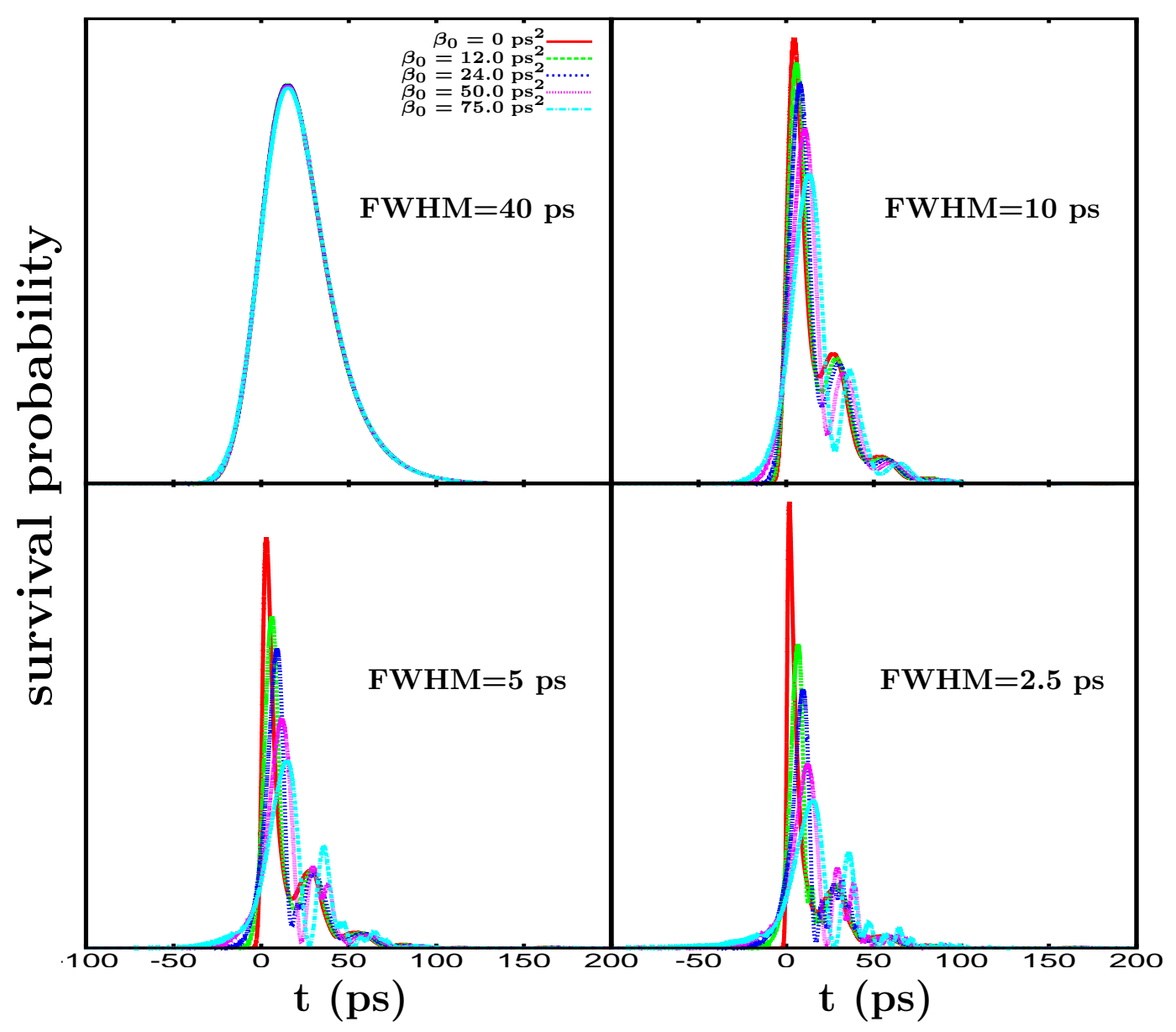


Fig. 3

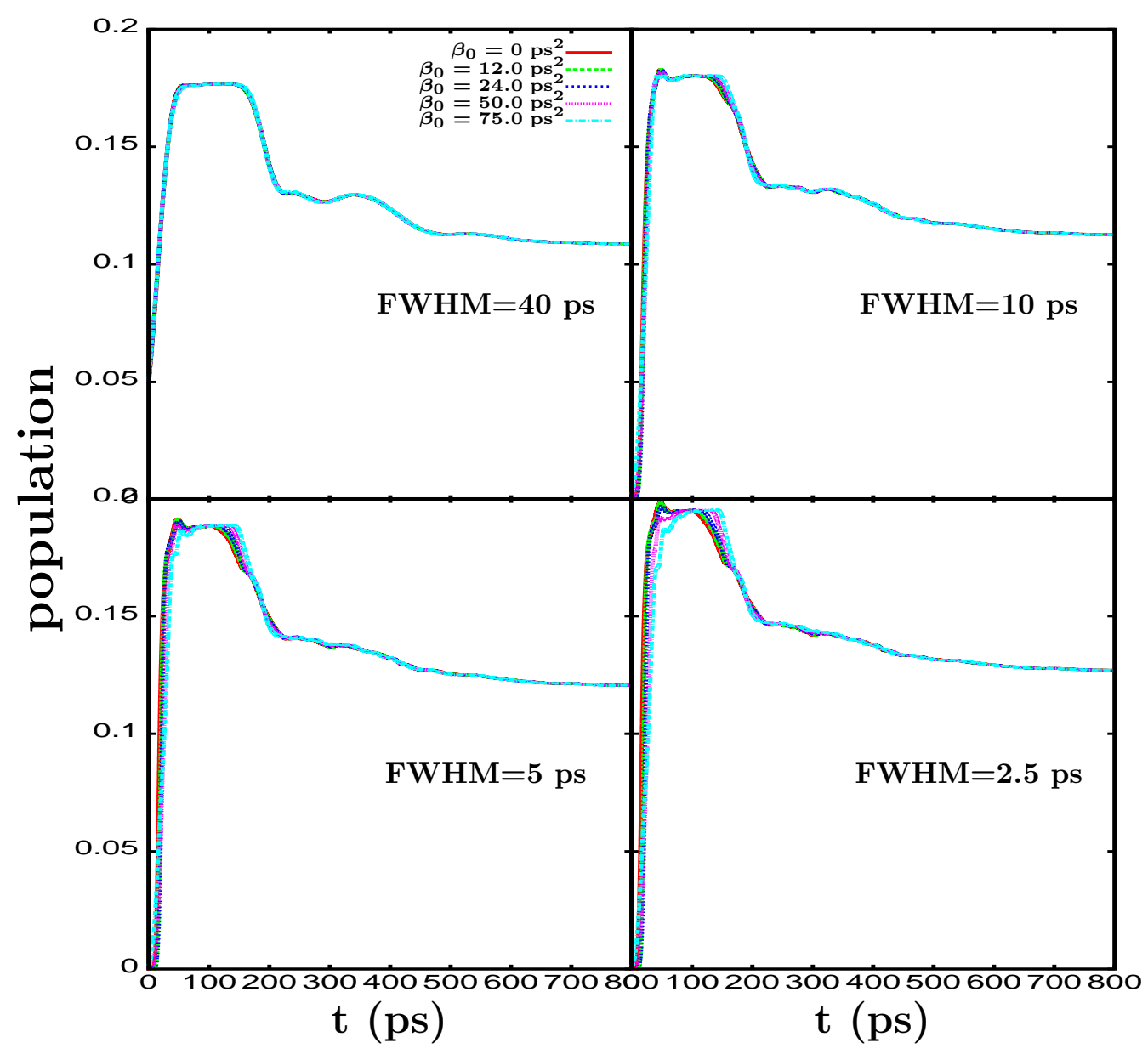


Fig. 4

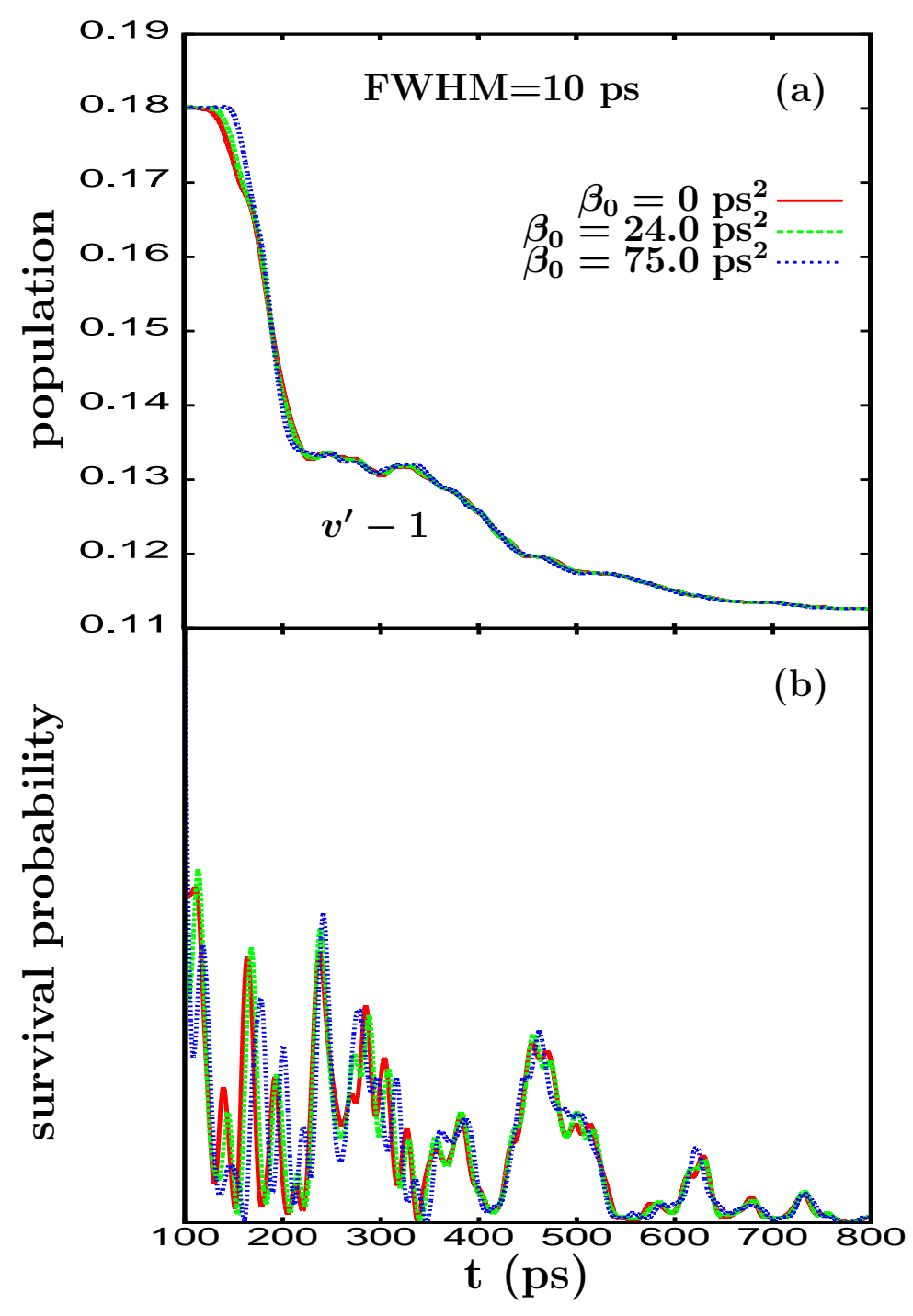


Fig. 5

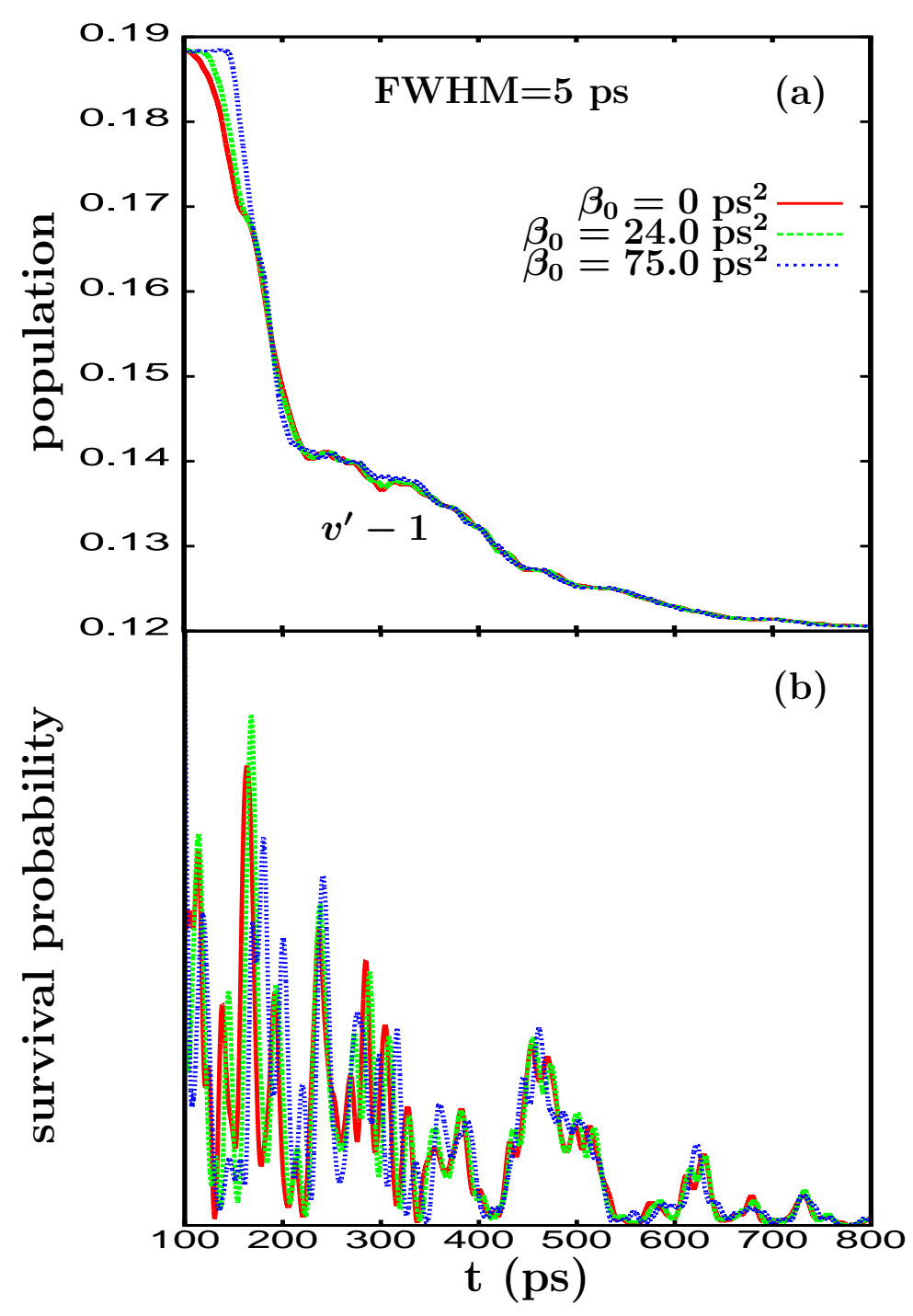


Fig. 6

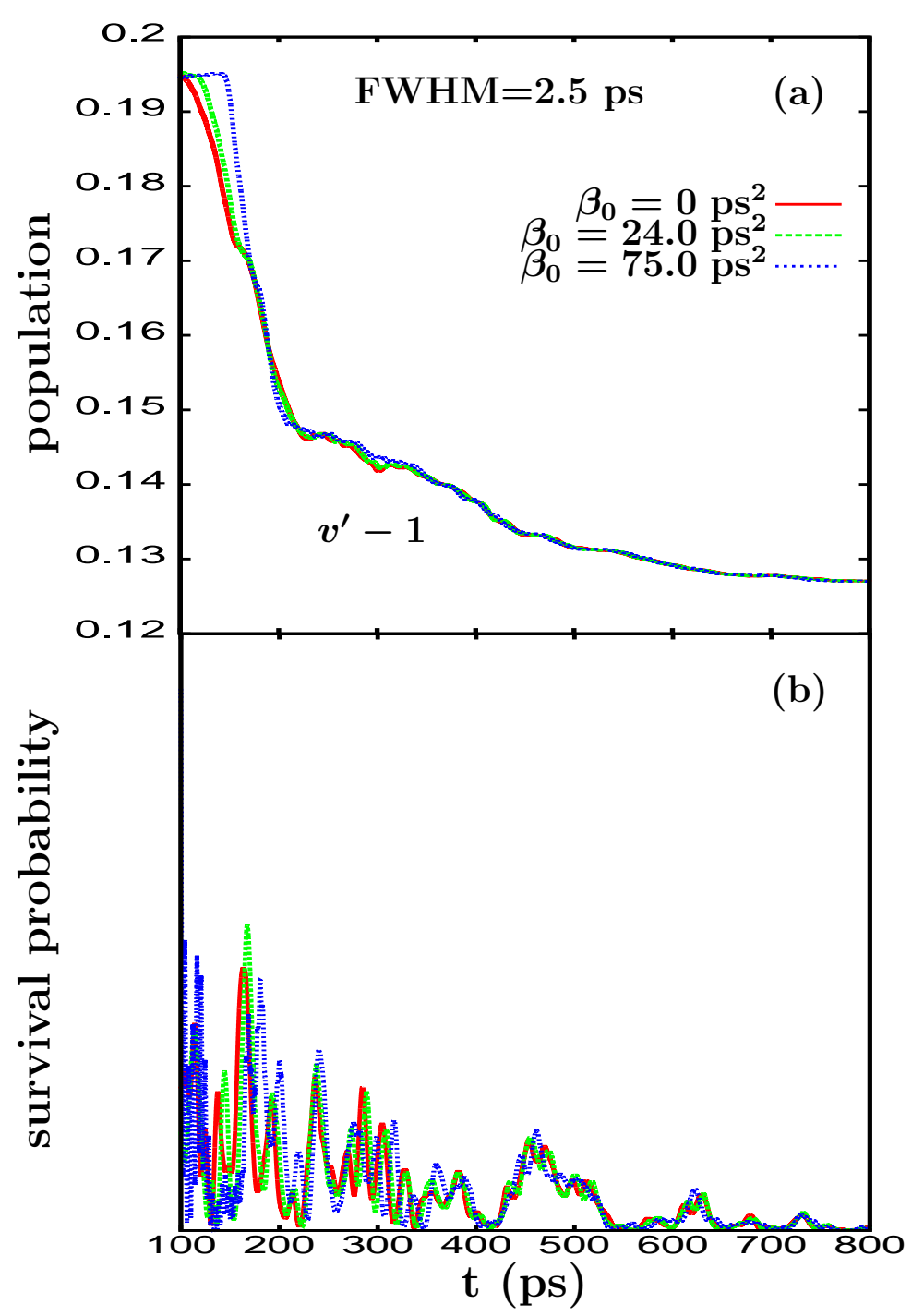

